\title{
Comparison of standardized methods for suspended solid concentration measurements in river samples
}

\author{
Guillaume Dramais, ${ }^{1,}$, Benoît Camenen ${ }^{1}$, Jérôme Le Coz ${ }^{1}$, Fabien Thollet $^{1}$, \\ Chloé Le Bescond ${ }^{1}$, Mickaël Lagouy ${ }^{1}$, Alexis Buffet ${ }^{1}$, Fréderic Lacroix ${ }^{1}$ \\ ${ }^{1}$ Irstea Lyon-Villeurbanne, RiverLy Research Unit, 69625 Villeurbanne, France
}

\begin{abstract}
SSC (Suspended Solid Concentration) measurements in rivers are a complex scientific issue. Many questions arise on the spatial and temporal distribution of particles throughout a cross-section, on the properties of particles and grain-size, and also on the sediment transport capacity of streams and rivers. In this study, we focused on the SSC and grain size distribution measured from river samples, automatically or manually acquired. Many agencies suggested slightly different methods for measuring SSC: The European standard NF EN 872, which related to the US EPA 160.2 requires sub-sampling using shake-and-pour aliquot selection. The APHA 2540D requires sub-sampling by pipetting at middepth in the original sample shaken with a magnetic stirrer. These methods lead to significant uncertainty when particles larger than $63 \mu \mathrm{m}$ are present in the samples. The ASTM D3977 analysis method, endorsed by the USGS is more accurate to capture and quantify particles larger than $63 \mu \mathrm{m}$. In this study we confirm the sub-sampling problem in a large concentration range using a set of samples from an alpine river.
\end{abstract}

\section{Introduction}

\subsection{Overview}

Measurement of suspended solids concentration (SSC) in rivers is an issue for watercourse managers and scientists to understand processes such as: erosion and deposition in navigation channels, movements of pollutants, management of dams and protection structures, etc. SSC refers to all insoluble solids present in a liquid sample. They can be mineral materials (clays, silts, sands) and organic particles. Sand, which is a part of the SSC, is a mineral granular, material, resulting from the erosion of the watershed mainly. It can be transported in rivers as bedload or in suspension. According to several classifications [1], sand particle size is between $0.063 \mathrm{~mm}$ (silt) and $2 \mathrm{~mm}$ (gravel). Sand mobilization through the flow is generally intermittent, depending on its availability, on hydrodynamic conditions, on the shape and the nature of the riverbed. Furthermore, sand suspension is not homogeneously distributed in the cross-section; it is defined as graded suspension, with an increase of the concentration while

*Corresponding author: guillaume.dramais@irstea.fr 
approaching the riverbed. The graded suspension is differentiated from the washload, which transports through the river particles finer than sands (clays, silts), distributed or mixed homogeneously in the cross-section. While the graded suspension of sand is in balance with the local hydrodynamics, washload depends less on the local transport capacity of the flow than on the upstream sediment supply.

\subsection{Sampling techniques}

There is a large range of instruments to collect samples of the water-sediment mixture in a river [2], depending on the size and flow conditions of the river, the samplers and the deploying devices and the purpose of the study. Suspended sediment measurements can be made using both manual or automatic samplers, including open containers, vertical and horizontal cylinders, bottle samplers, pumping samplers, single-stage samplers, pointintegrating samplers and depth-integrating samplers. Samples are collected from river bank, bridge, boat at the surface or distributed in the cross section at different depths. Samples consist of a mixture of sediment and water, sometimes integrated in space or time or instantaneous. An open question is therefore the representativeness of a single sample but also of a set of distributed samples to describe the mean concentration and flux throughout a river section.

\subsection{Study objectives}

The collected samples, stored in bottles, are then analysed, for SSC, particle size distributions or chemical element concentration. Indeed, scientists are interested in knowing the SSC for different purposes such as: establishing relation between SSC and turbidity, computing pollutants or sediment fluxes, studying river and habitat dynamics. However, there are significant uncertainties in estimating the SSC depending on the methodology used, in particular because of the sub-sampling. Sub-sampling is a further step of the analysis method, which consists to separate the sample into two or more aliquots. This study first describes the different standards used to analyse the SSC and compares the analyses of a set of samples from an alpine river, in a wide range of concentration. Then, we will show the limits of the NF EN 872 standard [3], available in France and Europe. We will evaluate the errors and consequences of sub-sampling and try to find ways to reduce them. We will evaluate how the presence of sand (particle diameter $>63 \mu \mathrm{m}$ ) in the samples will influence the performances of the different methods.

\section{Standards methods}

\subsection{Overview}

We could find standards describing slightly different methods for solid-phase measurement in water samples $[3,4,5,6,7,8]$. At least six standardized methods are currently available. Fig. 1 summarizes the theoretical validity ranges and the main steps of the different standards. After the sampling, each standardized method describes different operating principles, with several points being common or particular to the different methods:

- These methods differ mainly in the preparation of the samples. Two choices are possible: using the complete sample or using a subsample.

- After the sample choice, the volume should be measured. All these standard methods (except the hydrometer, see Fig. 1) are based on the separation of the solid and liquid phases of the sample by filtration, evaporation or centrifugation. 
- After separation, drying and weighing steps are common in each protocol to determine the sediment dry weight for the considered volume. The important steps of sample conservation, filter preparation and cooling, which are common to all the approaches are not described in the Fig. 1.

\subsection{Sub-sampling operation}

The most popular sub-sampling method is the shake-and-pour method, which is common to NF EN 872 [3], EPA 160.2. [8] and ISO 4365 (B) [7] standards. It consists in shaking the sample vigorously and quickly pouring the aliquot in a container of known volume. Another method commonly used and describe in the APHA SM 2540D [5] standard, is to prepare the sub-sample with a pipette in the whole sample stirred with a magnetic stirrer.

\subsection{Separation of solid and liquid phases}

\subsubsection{Filtration method}

The European standard [3] describes the sub-sample filtration procedure through a fiberglass filter. The American standards APHA SM 2540D [5] and EPA 160.2 [8] also describe a filtration method. An international standard exists: ISO 4365 [7] it offers several options (A, $\mathrm{B}, \mathrm{C})$ for separating the liquid and solid phases depending on the concentration of the samples, but without specifying a range of validity. Option B describes the filtration with a through a paper filter or through a fiberglass filter. In the US, ASTM D3977 [6] standards, offers three phase separation options, two of them by filtration: the first (B) by filtration of the whole sample and the second $(\mathrm{C})$ which consists in separating the sand from the sample in a sieve before the filtration. This option (wet-sieving of coarser particles before filtration) is considered as a reference in this study, because we want to evaluate the amount of sand in each sample.

\subsubsection{Other methods}

The total evaporation of the sample water is a method proposed in the ISO 4365 (A) [7], and ASTM D3977-97 (A) [6] standards. This method was not tested in this study because we focused on the sub-sampling problem. The total evaporation methods make it possible to measure the SSC and are accurate methods, but they don't allow the measurement of the amount of sand in the sample.

The centrifugation is described in the French standard NF T90-105-2 [4]. It consists of separating the solid and liquid phases in a centrifuge device. It is recommended for highly concentrated samples to avoid filter clogging. 


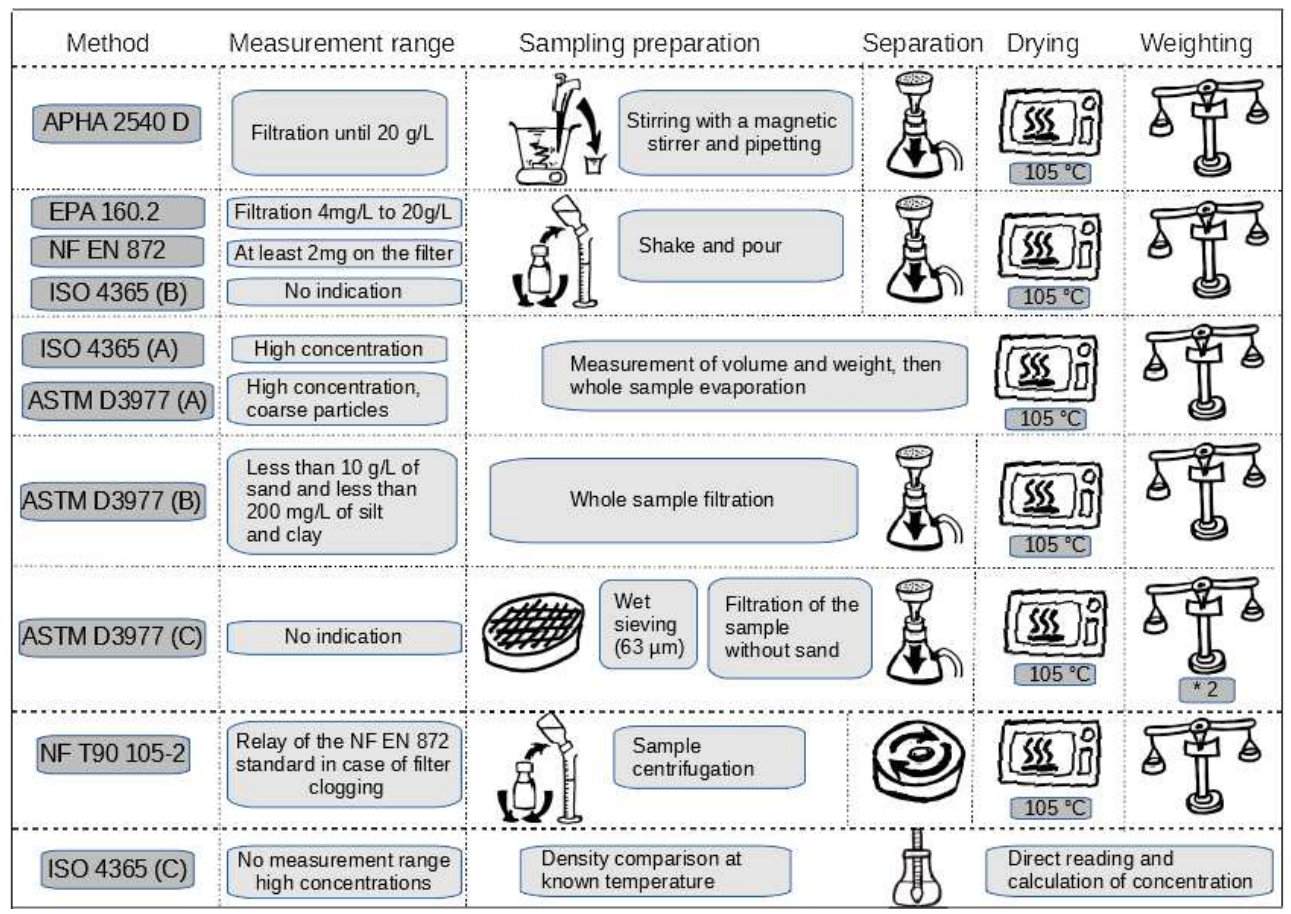

Fig. 1. Schematic representation of standardized protocols for sediment concentration measurements.

\subsection{American standards comparison}

US agencies elaborated the TSS (total suspended sediments) method, which was originally designed for wastewater analyses. In contrast, the SSC method endorsed by the USGS is supposed to provide reliable results for samples of natural water such as river samples. Several studies go that way like the works of Gray [9] conducted on 3,200 pairs of US river samples and Glysson [10] on 14,466 pairs of samples which compare both SSC and TSS methods, especially in the case of samples containing sand-size material. They noticed an underestimation of the sediment concentration (by 25-34\%) when sub-samplings were obtained by the shake and pour method or the pipette method versus the wet sieving method. Other authors such as Guo [11] and Clark [12,13] went further in the comparison and the investigation of sub-sampling effects. Selbig [14] contributed to this debate by studying the influence of grain size and the establishment of correction factors between SSC and TSS. They confirmed the limitations of TSS methods for measuring coarse particle concentration samples. These studies show the sensitivity of these methods. In Europe standardization agencies defined "suspended solid" in a way closer to TSS methods and thus avoiding the concerns of coarse particles and sub sampling. After an investigation in French laboratories involved in measuring the suspended sediment in rivers, we realized that sometimes they use the NF EN 872 [3] standard and sometimes they use methods to deal with the coarse particles issue influenced by American standards or others, but there is no general agreement. 


\section{Data set}

\subsection{Location}

The Arc-en-Maurienne River, is a tributary of the Isère River in the South East of France. This Alpine river is characterized by a nival hydrologic regime and an intense input of sediments from the catchment (approximately $2000 \mathrm{~km}^{2}$ ). Three run-of-the-river dams are managed by EDF (Electricité de France) which operates flushing every year in June. One hundred pairs of samples were collected during the 2017 flushing event with a specific pumping device, designed as a multi-depth sampler [15].

\subsection{Dataset}

The sample set was analyzed according to the ASTM [6] method. The dry samples contain $2.2 \%$ of organic matter on average, destroyed by exposure in a $500^{\circ} \mathrm{C}$ oven. This analyze was carry out on 35 samples. The samples have variable sand amounts ranging from $11 \%$ to $90 \%$ of the dry mass (Fig. 2). The main interest of this sample set is the broad range of concentration from less than $1 \mathrm{~g} / \mathrm{L}$ to more than $10 \mathrm{~g} / \mathrm{L}$. In the particular case of this flushing event, we observed a correlation between the suspended sediment concentrations and the percentage of sand in the samples. In high shear stress conditions, the sand is moving in suspension through the flow. And during flushing events there is more erosion in the dam's reservoirs, which results in high fine sediment concentration as well.

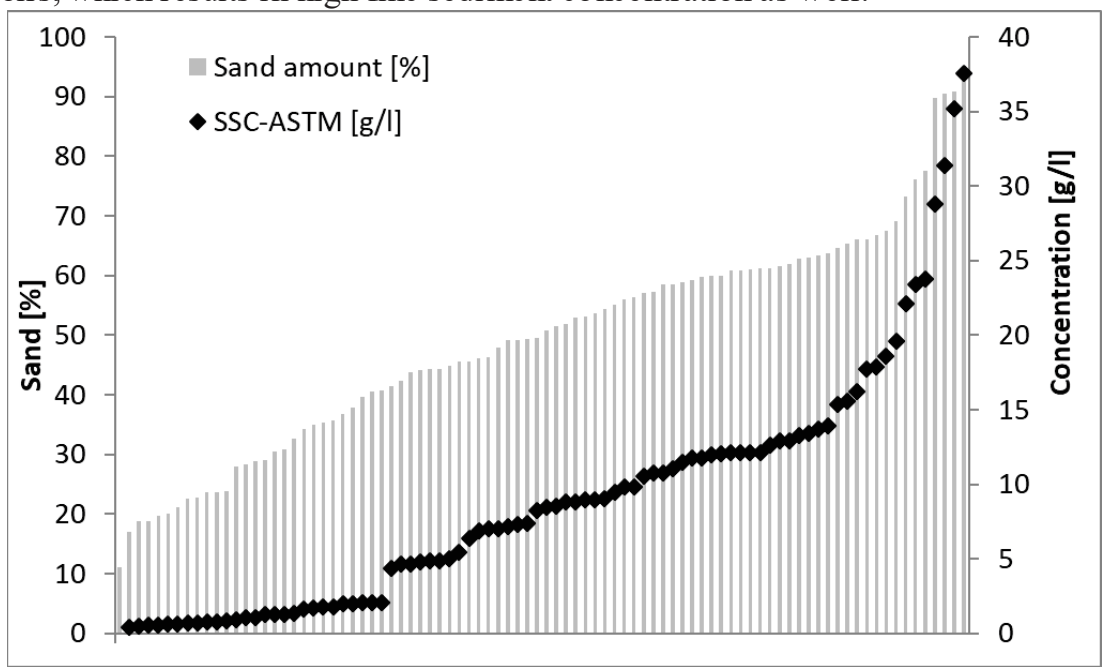

Fig. 2. Amount of sand (diameter $>63 \mu \mathrm{m}$ ) and SSC measured for the entire set of samples, (sorted by increasing concentrations).

\section{Results}

\subsection{Sub-sampling effect}

The comparison of 28 sample pairs measured with the ASTM (C) [6] method, i.e. separation of the sand by wet-sieving before filtration, versus the NF EN 872 [3] method, i.e. shake and pour subsampling method before filtration, is consistent with the conclusions found in the previous studies on US standards comparisons, i.e. a strong underestimation by the NF EN 
872 method (Fig. 3), in a wide range of concentration. Fig. $3 \mathbf{b}$ highlights one of the reasons for this underestimation: there is an important correlation between the amount of sand in the sample and the difference observed between the results of both analysis methods, especially when the percentage of sand is greater than 50\%. If we consider the ASTM [6] method as a reference, the NF EN 872 [3] method underestimates the concentration of samples with a large amount of sand. Errors ranging from $25 \%$ to over $50 \%$ are made for samples containing more than $40 \%$ of sand.

(a)

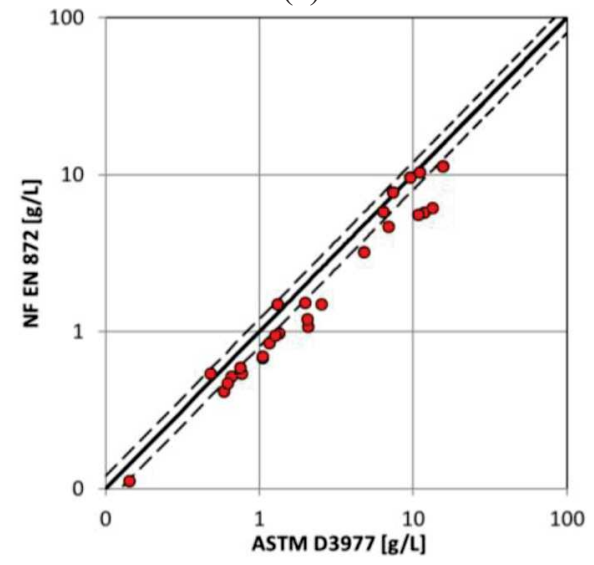

(b)

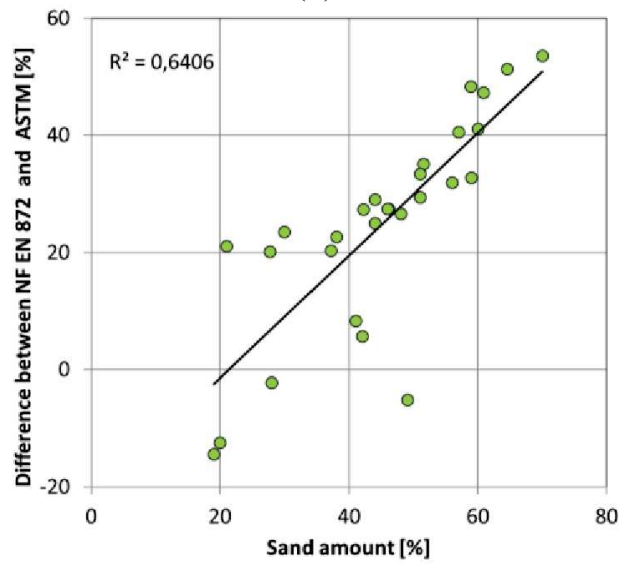

Fig. 3. (a) Comparisons between ASTM [6] and NF EN 872 [3] concentrations. The dotted lines are $\pm 20 \%$ envelopes, (b) differences calculated in percentage between both methods based on the percentage of sand.

In this comparison the shake and pour sub-sampling method is clearly the cause of the underestimation of the concentrations with the NF EN 872 [3] method, even for samples with relatively low concentration $(<1 \mathrm{~g} / \mathrm{L})$. Indeed, despite a vigorous agitation, taking a subsample from the sample container can result in undersampling sand sized particles because of their rapid settling before the aliquot can be obtained. The concentrations of four samples are nevertheless overestimated by the NF EN 872 [3] method. It is possible that the few grains of sand present in the sample may be trapped or not on the filter, consequently the sample concentration is overestimated or underestimated but biased either way.

Another method of subsampling is tested further on 35 pairs of various concentrations samples (Fig. 4) The magnetic stirring and pipetting method APHA [5] described in 2 is compared to the ASTM (C) [6] method. 
(a)

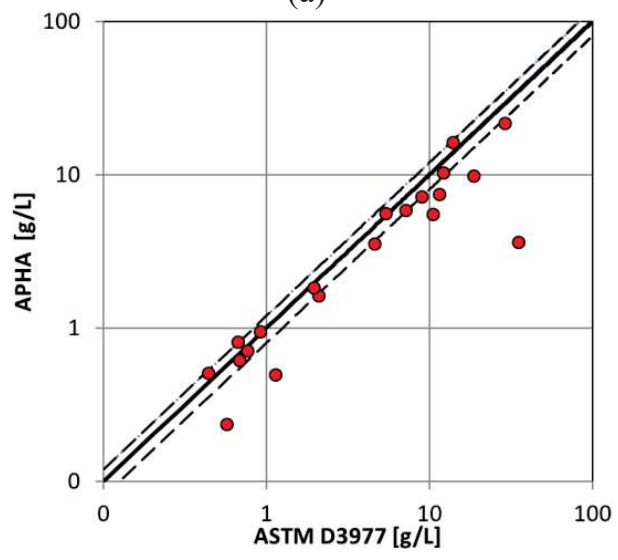

(b)

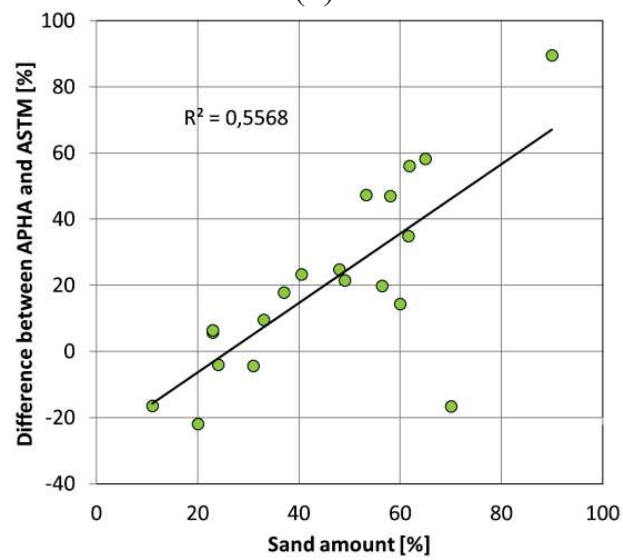

Fig. 4. (a) Comparisons between ASTM [6] and APHA [5] concentrations. The dotted lines are $\pm 20 \%$ envelopes, (b) differences calculated in percentage between both methods based on the percentage of sand.

In this comparison, results from the APHA [5] method with sub-sampling by pipetting are quite similar to the observations of the previous experiment with the NF EN 872 [3] standard. Underestimation of the concentration is almost proportional to the amount of sand particles present in the sample, five samples do not follow this trend. They are mostly low concentrated in fine and coarse particles.

\section{Conclusion}

This work conducted on a set of samples from the Arc-en-Maurienne River, collected in June 2017 in a broad range of concentrations (from 0.2 to $35 \mathrm{~g} / \mathrm{L}$ ) and for large sand amounts (from 10 to $90 \%$ ) confirms studies carried out in the United States on the limits of subsampling of river samples when coarse particles are included. The wet sieving method avoids the sediment settling problem during the subsampling. This study highlights several points of interest for scientists and managers interested in measuring the sediment concentration and particle size of river samples:

1. The method of the American standard ASTM D3977 [6] was used in this study as a reference because it is more likely to capture all the coarsest particles. It has the disadvantage of being time consuming but it is suited for the analysis of river water, especially in the presence of suspended sand and it can also provide the measure of sand concentration in the sample.

2. The presence of sand $(>63 \mu \mathrm{m})$ in samples defaults the shake and pour sub sampling method described in the NF EN 872 [3]. This has direct consequences (from 10\% to $60 \%$ underestimation) on the results of sediment concentration but also on other measurements. As an example, if this kind of sub-sampling is carried out to make a particle size analysis, the presence of the coarsest particles settling at the bottom of the bottle will be underestimated. The variants of subsampling methods with pipetting and magnetic stirring do not significantly reduce the errors, which remain large and highly variable.

Other sets of samples from the Rhône river were collected, the analysis of which confirms our present conclusions. In France and Europe it is important to consider this problem in order to improve sediment concentration measurements in rivers. It is important to elaborate 
or to choose the method best fitted to our needs, especially in flood conditions. This is to be able to compare the studies carried out on measuring the concentration of suspended sediment: flow budgets, turbidimeter calibration curves, concentrations of pollutants bound to particles and hydro-sedimentary studies.

We thank EDF-CIH for funding the field experiment during the 2017 dam flushing operation in the Arc River. This study was conducted within the Rhône Sediment Observatory (OSR), a multi-partner research program funded through the Plan Rhône by the European Regional Development Fund (ERDF), Agence de l'eau RMC, CNR, EDF and three regional councils.

\section{References}

1. F. Hjulström, Studies in the morphological activity of rivers as illustrated by the river Fyris. Bull. Geol. Inst. Uppsala 25, 221-527 (1935)

2. J.R. Gray, M.N. Landers, Measuring Suspended Sediment. In: Ahuja S. (ed.) Comprehensive Water Quality and Purification, vol. 1, pp. 157-204 (2014)

3. AFNOR. NF EN 872 Water quality - Determination of suspended solids - Method by filtration through glass fibre filters. (2005)

4. AFNOR. NF T90-105 Water quality - Determination of suspended solids - Method by centrifugation. (1997)

5. APHA. Standard methods for the examination of water and wastewater, 19th Ed., American Water Works Association and the Water Environment Federation, Washington, D.C. (2005)

6. ASTM. Standard test method for determining sediment concentration in water samples. D3977-97R07, West Conshohocken, PA. (2007)

7. ISO. 4365. Liquid flow in open channels - Sediment in streams and canals Determination of concentration, particle size distribution and relative density. (2005)

8. USEPA. Method 160.2: Total suspended solids (TSS) (gravimetric, dried at $103-105^{\circ} \mathrm{C}$ ), Revised Ed., Washington, D.C. (1999).

9. J. R. Gray, G. D. Glysson, L. M. Torcios, and G. Schwartz. Comparability of suspendedsediment concentration and total suspended solids data. Rep. WRIR 00-4191, U.S. Geological Survey Water-Resources Investigation, Reston, VA. (2000)

10. G. D. Glysson, J. R. Gray, Total suspended solids data for use in sediment studies. Turbidity and Other Sediment Surrogates Workshop, Reno, NV. (2002)

11. Q. Guo, Correlation of total suspended solids (TSS) and suspended sediment concentration (SSC) test methods. Final Rep. Prepared for New Jersey Department of Environmental Protection, Division of Science, Research, and Technology, Rutgers Dept. of Civil and Environmental Engineering, Piscataway, NJ. (2006)

12. S. E. Clark, C. S. Siu, Measuring solids concentration in stormwater runoff: Comparison of analytical methods. Environ. Sci. Technol., 42(2). (2008)

13. S. E. Clark, R. Pitt, Comparison of stormwater solids analytical methods for performance evaluation of manufactured treatment devices. Journal of Environmental Engineering, 137 (11). (2011)

14. W.R. Selbig, R.T. Bannerman, Ratios of total suspended solids to suspended sediment concentrations by particle size. Journal of Environmental Engineering, 137 (11). (2011)

15. B. Camenen, G. Dramais, A. Buffet, F. Thollet, C. Le Bescond, M. Lagouy, C. Berni, J. Le Coz, Estimation of sand suspension in a secondary channel of an alpine river. River Flow conference, Lyon. (2018) 